\title{
GRADE IV HYPERTENSIVE RETINOPATHY AS A RARE FIRST PRESENTATION OF HYPERTENSION: A CASE REPORT
}

\author{
Tengku Kamalden TA, Choo MM and Fauzi A \\ Department of Ophthalmology, Faculty of Medicine, University of Malaya, 50603 Kuala Lumpur, Malaysia
}

\begin{abstract}
Malignant hypertension affects less than 1\% of people with high blood pressure, and is a hypertensive emergency. It is rare for patients to present initially with this form of elevated blood pressure, which is almost always associated with acute target organ damage, which can manifest in many forms including ocular, neurological, cardiac and renal. This treatable condition is associated with a high rate of morbidity and mortality therefore, early detection and immediate management is of paramount importance. (JUMMEC 2006; 9(2): 32-34)
\end{abstract}

KEYWORDS: Malignant hypertension, hypertensive emergency, mortality

\section{Introduction}

Hypertension is a major risk factor for the development of serious complications affecting the heart, brain, kidneys, eyes and the vascular system, leading to death and disability on a huge scale globally. As more and more Malaysians are diagnosed with hypertension every year, it is important for clinicians to be aware of the acute and chronic changes seen in a fundoscopic examination.

\section{Case Report}

A 45 year old previously well Malay man presented with gradual blurring of vision in both eyes over a period of two weeks. This was preceded by six weeks of intermittent occipital headaches which were aggravated by eye straining to focus when reading. There was no nausea or vomiting. He denied any limb weakness or numbness.

On examination, visual acuity was OD 6/12, ph 6/12 and $O S 2 / 60$. The anterior segment examination was normal for both eyes, with no relative afferent papillary defect. Dilated fundus examination showed bilateral papilloedema with splinter haemorrhages temporal to the disc in the left eye and multiple cotton-wool spots at the posterior pole (Figure I). A macular star and mild macular oedema was also noted, which explained the poor left vision. There was narrowing of the arterioles with arteriovenous nipping. His blood pressure was recorded as $230 / 130$ $\mathrm{mmHg}$ with a pulse rate of 84 beats per minute. Neurological and cardiovascular assessments were normal. The rest of the systemic examination was unremarkable.

He was diagnosed as having malignant hypertension with Grade IV hypertensive retinopathy and choroidopathy. He was referred to the physicians for urgent management of hypertensive crisis. His blood pressure was reduced by titration of isosorbide dinitrate infusion to maintain a reading of lower than 160/90 $\mathrm{mmHg}$. Renal function tests showed renal failure with elevated serum creatinine and urea levels of $16.6 \mathrm{mmol} / \mathrm{l}$ and $732 \mu \mathrm{mol} / \mathrm{l}$ respectively. Urine examination showed gross proteinuria at $5 \mathrm{~g} / \mathrm{l}$, and 24-hour urine protein was markedly elevated with a reading of $1.8 \mathrm{~g}$. An ultrasonography of the kidneys showed bilateral small kidneys which measured $7.60 \mathrm{~cm}$ and $8.05 \mathrm{~cm}$ in bipolar length in the right and left kidneys, respectively. This was suggestive of bilateral renal parenchymal disease. All these evidence confirmed the presence of chronic renal failure.

\footnotetext{
Correspondence:

Dr Tengku Ain Fathlun Tengku Kamalden

Department of Ophthalmotology

Faculty of Medicine

University of Malaya

50603 Kuala Lumpur, Malaysia

Email: ain@ummc.edu.my
} 

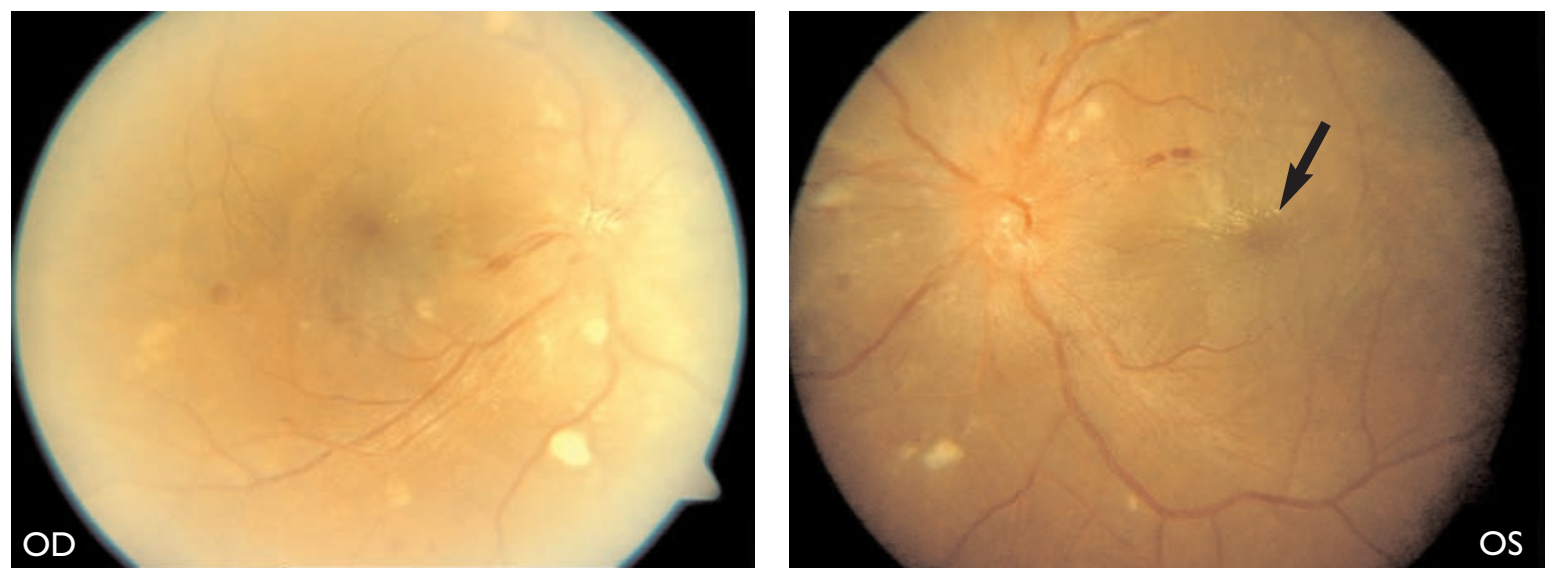

Figure I. The right fundus showed multiple cotton-wool spots with papilloedema. There was an early macular star in the left eye as shown by the black arrow. (OD: right eye; OS: left eye)

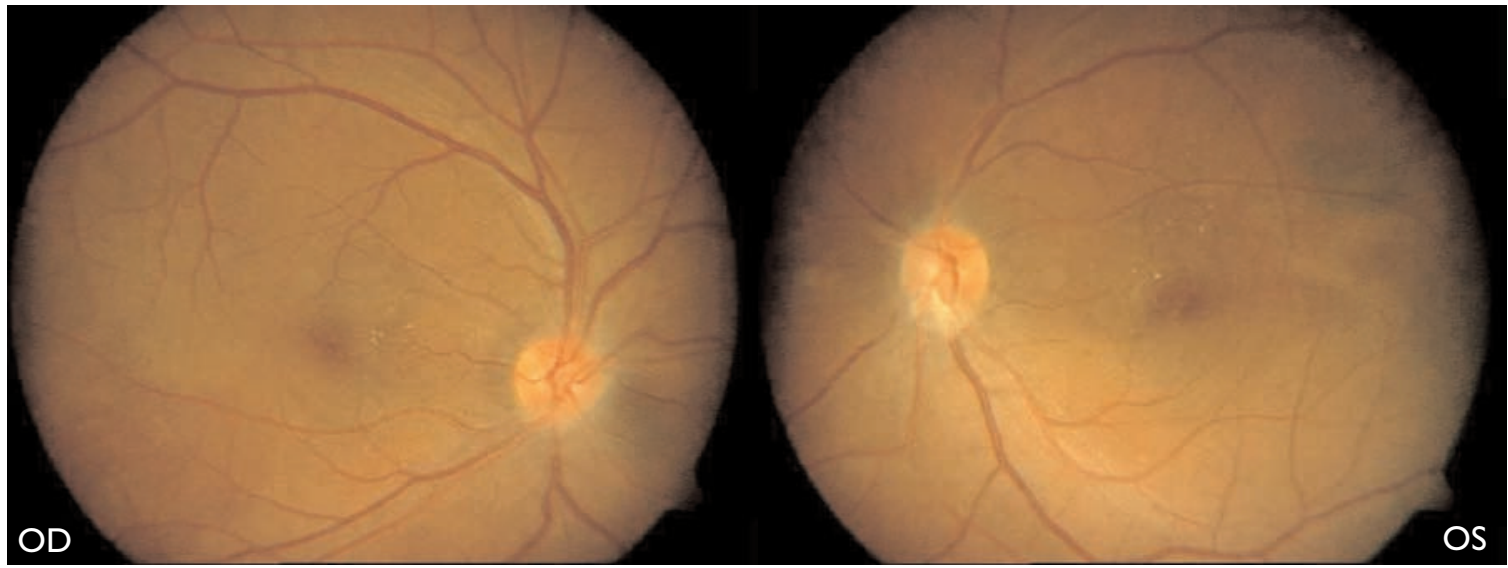

Figure 2. Fundus photos taken two months later showed almost complete resolution of the hypertensive retinopathy and choroidopathy changes with minimal residual hard exudates at both macula.

His blood pressure normalized to $130 / 80 \mathrm{mmHg}$ by the third day of admission, and his left vision improved to OS $6 / 18$. He was counseled regarding renal failure and the future need for dialysis. He was subsequently discharged with oral anti-hypertensive agents and iron supplements.

Three months following his discharge, his vision improved to a score of $6 / 9$ in both eyes, and fundus examination showed complete resolution of the hypertensive changes and papilloedema (Figure 2). By the eighth month following the first presentation to the eye clinic, his best corrected vision recovered fully to $6 / 6$ in both eyes. There was no evidence of optic atrophy in either eye.

\section{Discussion}

Malignant hypertension, or accelerated hypertension, is a rare syndrome consisting of rapid and severe elevation of blood pressure with the systolic component above $200 \mathrm{mmHg}$ or the diastolic blood pressure greater than $140 \mathrm{mmHg}$. The presence of systemic findings defines malignant hypertension. These include ocular, cardiac, renal and cerebral injury. Persistent malignant hypertension can lead to a rapidly fatal course with heart failure, myocardial infarction, stroke or renal failure.

Besides retinopathy, hypertensive choroidopathy may also occur, causing serious detachment, Elshnig's spots 
and Siegrist's streaks. These changes are caused by late leakage of choroidal vessels and focal necrosis of the retinal pigment epithelium. Cotton-wool spots usually resolve by 3-6 weeks, but arteriolar narrowing and AV nipping changes are permanent (I).

Immediate management of malignant hypertension is aimed at lowering the blood pressure in a controlled fashion to a level that minimises end-organ damage. Too rapid a decline can lead to ischaemia of the optic nerve head, brain and other vital organs, resulting in permanent damage. Treatment in this patient was initiated in a controlled, monitored setting under the close supervision of a physician.

With appropriate clinical diagnosis, hypertensive emergencies can be successfully treated and the complications can be prevented with timely intervention (2). From a systemic viewpoint, the diagnosis of a malignant hypertensive crisis represents a medical emergency. Untreated, it has a high mortality rate of
$50 \%$ at two months and $90 \%$ at one year (I). Thus early recognition of signs and symptoms, and early diagnosis is imperative to prevent fatalities.

The visual prognosis in malignant hypertension is good, with most patients resuming normal vision. However, on the rare occasion of visual loss, this may result from retinal pigment changes secondary to retinal detachment or from optic atrophy due to prolonged papilloedema. The visual prognosis in this patient was good, with full recovery of vision over time, along with the control of his blood pressure.

\section{References}

I. Yanoff M, Duker J, Eds. Ophthalmology, 2nd ed. St Louis: Mosby, 1999; 849-53.

2. Tuncel M, Ram VC. Hypertensive emergencies: etiology and management. Am J Cardiovasc Drugs 2003; 3(I): 2|-3I. 